\title{
CONGRUENCES FOR RATIONAL POINTS ON VARIETIES OVER FINITE FIELDS
}

\author{
N. FAKHRUDDIN AND C. S. RAJAN
}

\begin{abstract}
We show that the number of rational points on the fibres of a proper morphism of smooth varieties over a finite field $k$ whose generic fibre has a "trival" Chow group of zero cycles is congruent to $1 \bmod |k|$. As a consequence we prove that there is a rational point on any degeneration of a smooth proper rationally chain connected variety over a finite field. We also obtain a generalisation of the Chevalley-Warning theorem.
\end{abstract}

\section{INTRODUCTION}

1.1. The main result of this paper is the following:

Theorem 1.1. Let $f_{i}: X_{i} \rightarrow Y, i=1,2$ be proper dominant morphisms of smooth irreducible varieties over a finite field $k$ and let $g: X_{1} \rightarrow X_{2}$ be a dominant morphism over $Y$. Let $Z_{i}$ be the generic fibre of $f_{i}$ and assume that $g_{*}: C H_{0}\left(Z_{1 \overline{k\left(X_{1}\right)}}\right)_{\mathbb{Q}} \rightarrow C H_{0}\left(Z_{2} \overline{k\left(X_{1}\right)}\right)_{\mathbb{Q}}$ is an isomorphism. Then for all $y \in Y(k)$,

$$
\left|f_{1}^{-1}(y)(k)\right| \equiv\left|f_{2}^{-1}(y)(k)\right| \bmod |k| \text {. }
$$

Specialising to the case $X_{2}=Y$ and $f_{2}=I d_{Y}$, we obtain:

Corollary 1.2. Let $f: X \rightarrow Y$ be a proper dominant morphism of smooth irreducible varieties over a finite field $k$. Let $Z$ be the generic fibre of $f$ and assume that $C H_{0}\left(Z_{\overline{k(X)}}\right)_{\mathbb{Q}}=$ $\mathbb{Q}$. Then for all $y \in Y(k),\left|f^{-1}(y)(k)\right| \equiv 1 \bmod |k|$.

When $Y=\operatorname{Spec}(k)$, the above corollary reduces to a theorem of H. Esnault [10]. Since we do not assume that $f$ is smooth, we are able to obtain congruences even for singular varieties.

An immediate consequence of Corollary 1.2 is that for $f: X \rightarrow Y$ as above, $|X(k)| \equiv$ $|Y(k)| \bmod |k|$. By taking $Y$ to be a point in Theorem 1.1 we obtain:

Corollary 1.3. Let $g: X_{1} \rightarrow X_{2}$ be a dominant morphism of smooth proper varieties over a finite field $k$. If $g_{*}: C H_{0}\left(X_{\overline{k(X)}}\right)_{\mathbb{Q}} \rightarrow C H_{0}\left(Y_{\overline{k(X)}}\right)_{\mathbb{Q}}$ is an isomorphism, then $|X(k)| \equiv|Y(k)|$ $\bmod |k|{ }^{1}$

Simple examples show that in contrast to Corollary 1.2, the assumption that $X_{1}$ and $X_{2}$ be proper cannot be omitted here.

The triviality of the Chow group of zero cycles of degree 0 , or even rational chain connectedness ${ }^{2}$ is not sufficient to guarantee the existence of a rational point for proper varieties

\footnotetext{
${ }^{1}$ This can also be proved using the method of 13 .

${ }^{2}$ We learnt of such an example, due to J. Kollár, from a talk by J. Iyer at the University of Essen in May 2003; but see Remark 3.4
} 
over finite fields which are not smooth. However, using alterations, we give a criterion for the existence of rational points which can be applied to all degenerations of smooth rationally chain connected varieties.

Corollary 1.4. Let $f: X \rightarrow Y$ be a proper dominant morphism of irreducible varieties over a finite field $k$ with $Y$ smooth. Let $Z$ be the generic fibre of $f$ and assume that $Z$ is smooth and $C H_{0}\left(Z_{\overline{k(X)}}\right)_{\mathbb{Q}}=\mathbb{Q}$. Then for any $y \in Y(k), f^{-1}(y)(k) \neq \emptyset$.

The corollary below generalises the Chevalley-Warning theorem [12, which is the special case $P=\mathbb{P}^{n}$ and $L_{i}=\mathcal{O}\left(d_{i}\right)$ with $\sum d_{i} \leq n$.

Corollary 1.5. Let $P$ be a smooth projective geometrically irreducible variety over a finite field $k$. Let $L_{1}, \cdots, L_{r}$ be very ample line bundles on $P$ such that $\left(K_{P} \otimes L_{1} \otimes \cdots \otimes L_{r}\right)^{-1}$ is ample, where $K_{P}$ is the canonical bundle of $P$. For $i=1,2, \ldots, r$, let $s_{i} \in H^{0}\left(P, L_{i}\right)$. Then

$$
\left|\left\{x \in P(k) \mid s_{i}(x)=0, i=1,2, \ldots, r\right\}\right| \equiv 1 \quad \bmod |k|
$$

Note that the congruence formula of Katz [], when it applies, only gives congruences modulo $p=\operatorname{char}(k)$. It would be interesting to know whether an analogue of the Ax-Katz theorem [14] holds in the above situation or whether all low degree intersections as above over $C_{1}$ fields always have rational points.

Our proofs, as is Esnault's, are based on the method of decomposing the diagonal originated by Bloch, the Grothendieck-Lefschetz trace formula and Deligne's integrality theorem. The novelty of our approach lies in the use of proper correspondences which allows us to deal with non-proper $Y$; this is crucial for obtaining congruences for (or even just existence of) rational points on singular varieties. The main technical ingredient for this is the refined intersection theory of Fulton and MacPherson [11].

\section{Proper Correspondences}

2.1. In this section we introduce the concept of proper correspondences and prove some of its properties. ${ }^{3}$ Most of the proofs are simple modifications of those in Fulton's book [1] and we prove only what we need for later use; several other results in [11, Chapter 16] for (usual) correspondences have analogues for proper correspondences.

Definition 2.1. Let $X$ and $Y$ be smooth irreducible varieties over a field $k$. The group of proper correspondences from $X$ to $Y, P \operatorname{Corr}(X, Y)$, is the free abelian group generated by irreducible subvarieties $\Gamma \subset X \times Y$ which are proper over $Y$ modulo the subgroup generated by elements of the form

$$
\left\{\operatorname{div}(f) \mid f \in k(Z)^{*}, Z \subset X \times Y \text { irreducible and proper over } Y\right\} .
$$

This is clearly a graded abelian group; we shall grade it by dimension (lower indices) or codimension (upper indices) as is convenient. For a cycle $\Gamma$ (not necessarily irreducible) in the free abelian group as above we shall denote its class in $\operatorname{PCorr}(X, Y)$ by $[\Gamma]$.

\footnotetext{
${ }^{3}$ A. Nair has informed us that a variant of this definition has been considered, in a different context, by E. Urban in the preprint: Sur les représentations $p$-adiques associées aux représentations cuspidales de $G S p_{4} / \mathbb{Q}, 2001$.
} 
If $f: X \rightarrow Y$ is a proper morphism, then the graph of $f$ gives an element $\left[\Gamma_{f}\right]$ of $\operatorname{PCorr}(X, Y)$. We shall show below that proper correspondences induce maps on cohomology generalising the maps induced by proper morphisms.

2.2. We recall some properties of étale (co)homology and cycle class maps that we shall need. The main reference is [7, Exposés VI-IX]; we note that the quasi-projectivity hypotheses there can be removed using the methods of [11. The compatibility of refined intersection products and refined cycle class maps stated below can be deduced using the methods of [11, Chapter 19]; see also [3].

Let $K$ be an algebraically closed field and fix a prime number $l \neq \operatorname{char}(K)$. For a variety $X$ over $K$ let $H^{i}(X):=H_{e t}^{i}\left(X, \mathbb{Q}_{l}\right), H_{c}^{i}(X):=H_{c, e t}^{i}\left(X, \mathbb{Q}_{l}\right)$ and let $H_{Z}^{i}(X):=H_{Z, e t}^{i}\left(X, \mathbb{Q}_{l}\right)$ for $Z$ a closed subvariety of $X$. We also let $H_{i}(X)$ denote the locally finite (or Borel-Moore) homology $H_{i}^{e t}\left(X, \mathbb{Q}_{l}\right)$. For any of the above groups $H$, we denote by $H(n)$, for an integer $n$, the corresponding Tate twisted group.

For a cycle $\alpha=\sum_{i} a_{i} Z_{i}$, on a variety $X$, we denote by $|\alpha|$ the support $\cup_{i} Z_{i}$ of $\alpha$. The following properties are proved in the references cited above:

(1) (Projection formula) For any variety $X$ there are cap product maps $H^{i}(X) \otimes H_{j}(X) \rightarrow$ $H_{j-i}(X)$ such that if $f: X \rightarrow Y$ is a proper morphism, $u \in H^{i}(Y), v \in H_{j}(X)$ then $f_{*}\left(f^{*}(u) \cap v\right)=u \cap f_{*}(v)$ in $H_{j-i}(Y)$.

(2) For $Z$ an irreducible variety of dimension $n, H_{2 n}(Z)(-n)$ is one dimensional.

(3) For $\alpha$ a cycle of dimension $k$ on a variety $X$ the fundamental class defines a canonical element $c l(\alpha) \in H_{2 k}(|\alpha|)(-k)$. This maps to an element, also denoted by $c l(\alpha)$, in $H_{2 k}(X)(-k)$ which is zero if $\alpha=0 \in C H_{k}(X)$.

(4) For $X$ smooth of pure dimension $n$ there is a canonical isomorphism $H_{2 k}(|\alpha|)(-k) \cong$ $H_{|\alpha|}^{2 n-2 k}(X)(n-k)$, so we also get an element $c l(\alpha) \in H_{|\alpha|}^{2 n-2 k}(X)(n-k)$.

(5) For $f: X \rightarrow Y$ a morphism and $\alpha$ a cycle on $X$ of dimension $n$ whose support is proper over $Y, f_{*}(c l(\alpha))=\operatorname{cl}\left(f_{*}(\alpha)\right)$ in $H_{f(|\alpha|)}^{*}(Y)(-n)$.

(6) For $\alpha$ and $\beta$ cycles of pure dimension $k$ and $l$ respectively in a smooth irreducible variety $X$ of dimension $n$

$$
c l(\alpha \cdot \beta)=\operatorname{cl}(\alpha) \cup \operatorname{cl}(\beta) \in H_{|\alpha| \cap|\beta|}^{4 n-2(k+l)}(X)(n-k-l),
$$

where the product on the left is the refined intersection product.

2.3. Let $\operatorname{dim}(X)=n, \operatorname{dim}(Y)=m$ and let $\Gamma$ be a representative for an element of $\operatorname{PCorr}_{r}(X, Y)$. Then $\Gamma$ induces a linear map $\Gamma_{*}: H^{i}(X) \rightarrow H^{2 m-2 r+i}(Y)(m-r)$ as the composite of the sequence of linear maps:

$$
\begin{aligned}
& H^{i}(X) \stackrel{p_{X}^{*}}{\longrightarrow} H^{i}(X \times Y) \stackrel{\cdot c l(\Gamma)}{\longrightarrow} H_{|\Gamma|}^{i+2(n+m-r)}(X \times Y) \cong \\
& H_{2 r-i}(|\Gamma|)(-r) \stackrel{p_{Y *}}{\longrightarrow} H_{2 r-i}(Y)(-r) \cong H^{2 m-2 r+i}(Y)(m-r) .
\end{aligned}
$$

Since $X$ and $Y$ are smooth, by duality we also get maps $\Gamma^{*}: H_{c}^{i}(Y) \rightarrow H_{c}^{2 n-2 r+i}(X)(n-r)$. Now suppose that $k$ is a finite field and $K$ an algebraic closure of $k$. All the cohomology groups discussed above, for varieties over $K$ which are base changed from varieties over $k$, have a continuous action of $\operatorname{Gal}(K / k)$. The maps $\Gamma^{*}$ and $\Gamma_{*}$ are then compatible with the Galois action.

The following lemma implies that the maps $\Gamma_{*}$ and $\Gamma^{*}$ only depends on $[\Gamma] \in P \operatorname{Corr}(X, Y)$. 
Lemma 2.2. Let $\Gamma$ be as above. Suppose there exists a closed subvariety $Z \subset X \times Y$ such that $|\Gamma| \subset Z,[\Gamma]=0$ in $C H_{*}(Z)$ and $\left.p_{Y}\right|_{Z}: Z \rightarrow Y$ is proper. Then $\Gamma^{*}$ and $\Gamma_{*}$ are the zero maps.

Proof. Suppose $Z$ is a closed subvariety of $X \times Y$ such that the projection $\left.p_{Y}\right|_{Z}: Z \rightarrow Y$ is proper. It follows from the projection formula that $\Gamma_{*}$ can also be defined as,

$$
H^{i}(X) \stackrel{\left.p_{X}\right|_{Z} ^{*}}{\longrightarrow} H^{i}(Z) \stackrel{\cdot c l(\Gamma)}{\longrightarrow} H_{2 r-i}(Z)(-r) \stackrel{p_{Y_{*}}}{\longrightarrow} H_{2 r-i}(Y)(-r) \cong H^{2 m-2 r+i}(Y)(m-r),
$$

where $c l(\Gamma)$ is considered as an element in $H_{2 r}(Z)(-r)$. The lemma follows from the fact that $c l(\Gamma)=0$ in $H_{2 r}(Z)(-r)$. The statement for $\Gamma^{*}$ follows by duality.

2.4. Let $X, Y, Z$ be smooth irreducible varieties over $K$ and let $\left[\Gamma_{1}\right]$ (resp. $\left[\Gamma_{2}\right]$ ) be a proper correspondence from $X$ to $Y$ (resp. $Y$ to $Z$ ). Analogous to the definition of composition of correspondences [11, Chapter 16], we define $\left[\Gamma_{2}\right] \circ\left[\Gamma_{1}\right] \in \operatorname{PCorr}(X, Z)$ by

$$
\left[\Gamma_{2}\right] \circ\left[\Gamma_{1}\right]=\left[p_{X Z_{*}}\left(p_{X Y}{ }^{*}\left(\Gamma_{1}\right) \cdot p_{Y Z}{ }^{*}\left(\Gamma_{2}\right)\right)\right],
$$

where the $p$ 's denote the projection morphisms from $X \times Y \times Z$ and the product is the refined intersection product. Note that $p_{X Y}{ }^{*}\left(\Gamma_{1}\right) \cdot p_{Y Z}{ }^{*}\left(\Gamma_{2}\right)$ is a cycle which is well defined upto rational equivalence on $\left|\Gamma_{1}\right| \times_{Y}\left|\Gamma_{2}\right|$. Since $\left|\Gamma_{1}\right| \times_{Y}\left|\Gamma_{2}\right|$ is proper over $Z$, its image in $X \times Z$ is also proper over $Z$, so $\left[\Gamma_{2}\right] \circ\left[\Gamma_{1}\right]$ is a well defined element of $\operatorname{PCorr}(X, Z)$.

Lemma 2.3. Let $X, Y, Z$ and $\Gamma_{1}, \Gamma_{2}$ be as above. Then $\left(\Gamma_{2} \circ \Gamma_{1}\right)_{*}=\left(\Gamma_{2}\right)_{*} \circ\left(\Gamma_{1}\right)_{*}$ as maps from $H^{*}(X)$ to $H^{*}(Z)$ and $\left(\Gamma_{2} \circ \Gamma_{1}\right)^{*}=\left(\Gamma_{1}\right)^{*} \circ\left(\Gamma_{2}\right)^{*}$ as maps from $H_{c}^{*}(Z)$ to $H_{c}^{*}(X)$.

Proof. The key point is the compatibility of the refined cycle class maps with refined intersection products.

Let $a \in H^{*}(X)$. Then

$$
\begin{aligned}
\left(\Gamma_{2}\right)_{*} \circ\left(\Gamma_{1}\right)_{*}(a) & =p_{Z}^{Y Z}{ }_{*}\left(\operatorname{cl}\left(\Gamma_{2}\right) \cdot p_{Y}^{Y Z^{*}}\left(p_{Y}^{X Y}{ }_{*}\left(c l\left(\Gamma_{1}\right) \cdot p_{X}^{X Y^{*}}(a)\right)\right)\right) \\
& =p_{Z}^{Y Z}{ }_{*}\left(c l\left(\Gamma_{2}\right) \cdot p_{Y Z}^{X Y Z}{ }_{*}\left(p_{X Y}^{X Y Z^{*}}\left(\operatorname{cl}\left(\Gamma_{1}\right) \cdot p_{X}^{X Y^{*}}(a)\right)\right)\right) \\
& =p_{Z}^{Y Z}{ }_{*}\left(p_{Y Z}^{X Y Z}{ }_{*}\left(p_{Y Z}^{X Y Z^{*}}\left(\operatorname{cl}\left(\Gamma_{2}\right)\right) \cdot p_{X Y}^{X Y Z^{*}}\left(\operatorname{cl}\left(\Gamma_{1}\right) \cdot p_{X}^{X Y^{*}}(a)\right)\right)\right) \\
& \left.=p_{Z}^{Y Z}{ }_{*}\left(p_{Y Z}^{X Y Z}{ }_{*}\left(p_{Y Z}^{X Y Z^{*}}\left(\operatorname{cl}\left(\Gamma_{2}\right)\right) \cdot p_{X Y}^{X Y Z^{*}}\left(\operatorname{cl}\left(\Gamma_{1}\right)\right) \cdot p_{X}^{X Y Z^{*}}(a)\right)\right)\right) \\
& \left.=p_{Z}^{X Z}{ }_{*}\left(p_{X Z}^{X Y Z}{ }_{*}\left(p_{Y Z}^{X Y Z^{*}}\left(c l\left(\Gamma_{2}\right)\right) \cdot p_{X Y}^{X Y Z^{*}}\left(\operatorname{cl}\left(\Gamma_{1}\right)\right) \cdot p_{X}^{X Y Z^{*}}(a)\right)\right)\right) \\
& =p_{Z}^{X Z}{ }_{*}\left(c l\left(\Gamma_{2} \circ \Gamma_{1}\right) \cdot p_{X}^{X Z^{*}}(a)\right) \\
& =\left(\Gamma_{2} \circ \Gamma_{1}\right)_{*}(a)
\end{aligned}
$$

We use the projection formula several times along with compatibility of the cycle class map with smooth pullbacks, products and proper pushforwards.

2.5. The key technical result which allows us to deduce congruences from cycle theoretic information is the following:

Proposition 2.4. Let $X, Y, \Gamma$ be as above and assume that $m=n=r$. If $\operatorname{dim}\left(p_{X}(|\Gamma|)\right)<n$ then all the eigenvalues of (the geometric) Frobenius acting on $\Gamma^{*}\left(H_{c}^{i}(Y)\right) \subset H_{c}^{i}(X)$ are algebraic integers which are divisible by $|k|$.

Proof. Replacing $k$ by a finite extension does not affect the conclusion of the lemma, so without loss of generality we may assume that $\Gamma$ is a geometrically irreducible subvariety of 
$X \times Y$. Using the definition of $\Gamma_{*}$ as given in the proof of Lemma 2.2, $\Gamma_{*}$ is the composite of the following maps:

$$
H^{i}(X) \stackrel{p_{X}^{*}}{\longrightarrow} H^{i}(\Gamma) \longrightarrow H_{2 n-i}(\Gamma)(-n) \stackrel{p_{Y} \mid \Gamma_{*}^{*}}{\longrightarrow} H_{2 n-i}(Y)(-n) \cong H^{i}(Y),
$$

where we have used the hypothesis that $m=n=r$.

Suppose $\pi: \Gamma^{\prime} \rightarrow \Gamma$ is a proper dominant generically finite morphism with $\Gamma^{\prime}$ smooth and geometrically irreducible. Then the projection formula shows that we may replace $\Gamma$ with $\Gamma^{\prime}$ and $p_{X}\left(\right.$ resp. $\left.p_{Y}\right)$ with $p_{X} \pi\left(\operatorname{resp} . p_{Y} \pi\right)$ in the above without changing the image of the composite.

Let $W$ be the Zariski closure of $p_{X}(\Gamma)$ in $X$ and let $\operatorname{dim}(W)=t$. By the theorem of De Jong [4, 4.1] we may find $\Gamma^{\prime}$ as above, $\sigma: W^{\prime} \rightarrow W$ with $\sigma$ proper dominant generically finite and $W^{\prime}$ smooth, and $p: \Gamma^{\prime} \rightarrow W^{\prime}$ such that $p_{X} \pi=i_{W} \sigma p$, where $i_{W}: W \rightarrow X$ is the inclusion. Since $p_{X} \pi=\left(i_{W} \sigma\right) p$ and both $\Gamma^{\prime}$ and $W^{\prime}$ are smooth, it follows from the functoriality of pushforward maps that $\left(p_{X} \pi\right)_{*}=\left(i_{W} \sigma\right)_{*} p_{*}$. By the remarks of the previous paragraph we see that the image of $\Gamma_{*}$ is the same as that of the composite of the sequence:

$$
H^{i}(X) \longrightarrow H^{i}\left(W^{\prime}\right) \longrightarrow H^{i}\left(\Gamma^{\prime}\right) \longrightarrow H^{i}(Y) \text {. }
$$

Since $\Gamma^{\prime}$ and $W^{\prime}$ are smooth, by duality we get a factorisation of $\Gamma^{*}$ as a composite of maps,

$$
H_{c}^{j}(Y) \longrightarrow H_{c}^{j}\left(\Gamma^{\prime}\right) \longrightarrow H_{c}^{2(t-n)+j}\left(W^{\prime}\right)(t-n) \longrightarrow H_{c}^{j}(X) .
$$

By Deligne's integrality theorem [6], Exposé XXI, Corollaire 5.5.3, all the eigenvalues of Frobenius on $H_{c}^{*}\left(W^{\prime}\right)$ are algebraic integers. Since $n-t>0$ and the geometric Frobenius acts on $\mathbb{Q}_{l}(t-n)$ by $|k|^{n-t}$, the proposition follows.

\section{Proofs of the main Results}

Using the results of the previous section, we now give the proofs of the results stated in the introduction.

\section{1.}

Proof of Theorem 1.1. If $Y$ is not geometrically irreducible, then $Y(k)=\emptyset$ so there is nothing to prove. The hypothesis on the Chow group implies that the geometric connected components of $Z_{1}$ and $Z_{2}$ are in bijective correspondence. Since $X_{1}$ and $X_{2}$ are smooth, we can assume that $X_{1}, X_{2}$ and $Y$ are geometrically irreducible.

Let $W$ be an irreducible subvariety of $X_{1}$ which maps generically finitely and dominantly to $X_{2}$ and let $d$ be the degree of $W$ over $X_{1}$. Let $\Gamma_{g}$ be the graph of $g$ in $X_{1} \times_{k} X_{2}$, let $\Gamma_{W}$ be the transpose of the graph of $\left.g\right|_{W}$ embedded in $X_{2} \times_{k} X_{1}$ and let $\Gamma_{1}=\left(\Gamma_{W} \circ \Gamma_{g}\right) / d$. Since $W$ is a subvariety of $X_{1}, \Gamma_{W}$ is a proper correspondence, and $\Gamma_{g}$ is a proper correspondence since $g$ is proper. Hence $\Gamma_{1}$ is a proper correspondence of dimension $n_{1}=\operatorname{dim} X_{1}$.

Let $V_{2}$ be the open subset of $X_{2}$ over which $\left.g\right|_{W}$ is finite and let $V_{1}=g^{-1}\left(V_{2}\right)$. By the construction of $W, p_{12}^{*}\left(\Gamma_{g}\right)$ and $p_{23}^{*}\left(\Gamma_{W}\right)$, which are subvarieties of $X_{1} \times_{k} X_{2} \times_{k} X_{1}$, meet properly when pulled back to $V_{1} \times_{k} X_{2} \times_{k} X_{1}$. It follows that one can write $\Gamma_{1}=\Gamma_{1}^{\prime}+\Gamma_{1}^{\prime \prime}$ in $P \operatorname{Corr}\left(X_{1}, X_{1}\right)$ where $\left(I d_{X_{1}} \times g\right)_{*}\left(\Gamma_{1}^{\prime}\right)=\Gamma_{g}$ in $\operatorname{PCorr}\left(X_{1}, X_{2}\right)$ and $p_{1}\left(\left|\Gamma_{1}^{\prime \prime}\right|\right)$ is a proper subvariety of $X_{1}$.

Let $\Delta_{X_{1}}$ be the diagonal in $X_{1} \times_{k} X_{1}$ and consider the proper correspondence $\Gamma_{2}:=\Delta_{X_{1}}-\Gamma_{1}$ and its restriction (i.e. flat pullback) $\gamma_{2}$ to $Z_{1} \times_{k(Y)} Z_{1}$. Since $\left(I d_{X_{1}} \times g\right)_{*}\left(\Delta_{X_{1}}\right)=\Gamma_{g}$, it follows from the previous paragraph that $\left(I d_{Z_{1}} \times\left. g\right|_{Z_{1}}\right)_{*}\left(\gamma_{2}\right)$ can be represented by a cycle on $Z_{1} \times_{k(Y)} Z_{2}$ which becomes zero when restricted to $k\left(Z_{1}\right) \times_{k(Y)} Z_{2}$. Since the map 
$g_{*}: C H_{0}\left(Z_{1} \overline{k\left(X_{1}\right)}\right)_{\mathbb{Q}} \rightarrow C H_{0}\left(Z_{2} \overline{k\left(X_{1}\right)}\right)_{\mathbb{Q}}$ is injective, it follows that $\gamma_{2}$ can be represented by a cycle on $Z_{1} \times_{k(Y)} Z_{1}$ whose support maps to a proper subvariety of $Z_{1}$ by the projection to the first factor. By taking the Zariski closures in $X_{1} \times_{Y} X_{2}$, it follows that $\Gamma_{2}$ can be represented by a proper correspondence on $X_{1} \times_{k} X_{1}$ whose support maps to a proper subvariety of $X_{1}$ by the projection to the first factor.

By Proposition 2.4 all the eigenvalues of Frobenius acting on the image of $\Gamma_{2}^{*}$ in $H_{c}^{*}\left(X_{1}\right)$ are divisible by $|k|$. It follows from Lemma 2.3 and the definition of $\Gamma_{1}$, that the image of $\Gamma_{1}^{*}$ is contained in the image of $g^{*}$. By the definition of $\Gamma_{2}$, we conclude that all the eigenvalues of Frobenius acting on the cokernel of $g^{*}: H_{c}^{*}\left(X_{2}\right) \rightarrow H_{c}^{*}\left(X_{1}\right)$ are divisible by $|k|$.

The hypotheses of the theorem, and the above discussion, are not affected if we replace $Y$ by an open subvariety $U$ and $X_{i}$ by $f_{i}^{-1}(U), i=1,2$, so we may assume that $Y$ has only one rational point $y$. Applying the Grothendieck-Lefschetz trace formula and the statement on eigenvalues above, we conclude that the $\left|X_{1}(k)\right| \equiv\left|X_{2}(k)\right| \bmod |k|$. Each rational point of $X_{i}$ must lie over the unique rational point $y$ of $Y$, hence the theorem follows.

Corollary 3.1. Let $f: X \rightarrow Y$ be a proper dominant generically finite morphism of smooth irreducible varieties over a finite field $k$ such that the extension of function fields $k(Y) \rightarrow$ $k(X)$ is purely inseparable. Then for any $y \in Y(k),\left|f^{-1}(y)(k)\right| \equiv 1 \bmod |k|$.

Proof. Since $\left(Z_{\overline{k(X)}}\right)_{r e d}$ is isomorphic to $\operatorname{Spec}(\overline{k(X)})$, the hypothesis on $C H_{0}$ is trivially satisfied.

Remark 3.2. Since we only use intersection theory (resp. cohomology groups) with rational (resp. $\mathbb{Q}_{l}$ ) coefficients, the above results hold even when $X$ and $Y$ are quotients of smooth varieties by finite groups.

\section{2 .}

Proof of Corollary 1.4. As in the proof of Theorem 1.2, we may assume that $X$ and $Y$ are geometrically irreducible and that $Y$ has a unique rational point.

By a result of De Jong [5, 5.15] there exists an irreducible variety $X^{\prime}$ over $k$ which is the quotient of a smooth variety by a finite group and a proper dominant generically finite morphism $\pi: X^{\prime} \rightarrow X$ such that the extension of function fields $k(X) \rightarrow k\left(X^{\prime}\right)$ is purely inseparable. Let $f^{\prime}=f \pi: X^{\prime} \rightarrow Y$ and let $Z^{\prime}$ be the generic fibre of $f^{\prime}$. Since $X^{\prime}$ is irreducible over $k, Z^{\prime}$ is irreducible over $k(Y)$. $Z$ is geometrically irreducible since it is smooth over $k(Y)$; since the extension of function fields induced by the map $Z^{\prime} \rightarrow Z$ is $k(X) \rightarrow k\left(X^{\prime}\right), Z^{\prime}$ is also geometrically irreducible. The induced morphism $\left(Z^{\prime} \overline{k(X)}\right)_{r e d} \rightarrow Z \overline{k(X)}$ thus satisfies the assumptions of Lemma 3.5 below, so $C H_{0}\left(Z^{\prime} \overline{k\left(X^{\prime}\right)}\right)_{\mathbb{Q}}=\mathbb{Q}$. Applying Corollary 1.2 (cf. Remark [3.2) to the morphism $f^{\prime}: X^{\prime} \rightarrow Y$ we see that $X^{\prime}(k) \neq \emptyset$. Thus $X(k) \neq \emptyset$.

Remark 3.3. For singular $X$ it is not always true that $\left|f^{-1}(y)(k)\right| \equiv 1 \bmod |k|$, however the only examples we know where this fails are non-normal.

Remark 3.4. $X(k) \neq \emptyset$ if $X$ is a proper variety over a finite field $k$ which is rationally connected i.e. any two general points of $X(\Omega)$ are contained in an irreducible rational curve in $X$ defined over $\Omega$, where $\Omega \supset k$ is a universal domain. (To prove this we may replace $X$ by $X^{\prime}$ as in the proof of Corollary 1.4. Since the extension of function fields $k(X) \rightarrow k\left(X^{\prime}\right)$ is purely inseparable, it follows that $X^{\prime}$ is also rationally connected, so $C H_{0}\left(X^{\prime} \overline{k\left(X^{\prime}\right)}\right)_{\mathbb{Q}}=\mathbb{Q}$. By Remark 3.2 it follows that $X^{\prime}(k) \equiv 1 \bmod |k|$, hence $X^{\prime}(k) \neq \emptyset$. Thus $X(k) \neq \emptyset$.) 
However, a degeneration of a rationally connected variety is in general only rationally chain connected, so one cannot use this to prove Corollary 1.4 even if $Z$ is rationally connected.

Lemma 3.5. Let $f: X \rightarrow Y$ be a proper dominant morphism of irreducible varieties over an algebraically closed field $K$. Assume that $Y$ is smooth, $f$ is generically finite and the extension of function fields $K(Y) \rightarrow K(X)$ is purely inseparable. Then $f_{*}: C H_{0}(X)_{\mathbb{Q}} \rightarrow C H_{0}(Y)_{\mathbb{Q}}$ is an isomorphism.

Proof. $f_{*}$ is surjective because $f$ is surjective. Using the refined intersection theory of [1] and the hypothesis on $Y$, we see that there is a natural map $f^{*}: C H_{0}(Y)_{\mathbb{Q}} \rightarrow C H_{0}(X)_{\mathbb{Q}}$. By the assumptions on $f$ there exists an open subset $U$ of $Y$ such that for $V=f^{-1}(U)$, $\left.f\right|_{V}: V \rightarrow U$ is a bijection. By the moving lemma (which is trivial for zero cycles) $C H_{0}(X)$ (resp. $C H_{0}(Y)$ ) is generated by the closed points in $V$ (resp. $U$ ). This shows that $f^{*}$ is a surjection since for $y \in U, f^{*}([y])=\left[f^{-1}(y)\right]$. Since $f_{*} f^{*}$ is multiplication by $\operatorname{deg}(f)$, it follows that $f_{*}$ is an isomorphism.

\section{3.}

Proof of Corollary 1.2. Let $Y=\prod_{i} H^{0}\left(P, L_{i}\right)$ and let $X \subset Y \times P$ be the zero scheme of the universal section of $\bigoplus_{i} p_{P}^{*} L_{i}$. Since the $L_{i}$ are basepoint free, the map $X \rightarrow P$ is smooth, hence $X$ is also smooth. Since the $L_{i}$ are assumed to be very ample, Bertini's theorem implies that the generic fibre $Z$ of the projection $f: X \rightarrow Y$ is smooth. The assumptions on the $L_{i}$ and the adjunction formula imply that $Z$ is a Fano variety. By a theorem of Campana 2] and Kollar-Miyaoka-Mori 15] $Z$ is rationally chain connected, so $C H_{0}\left(Z_{\overline{k(X)}}\right)_{\mathbb{Q}}=\mathbb{Q}$. The proof is concluded by applying Corollary 1.2 to $f$.

Remark 3.6. The proof shows that instead of assuming that the $L_{i}$ are very ample it suffices to assume that they are basepoint free and that $Z$ is smooth.

A motivic version of the above corollary in the case of hypersurfaces in $\mathbb{P}^{n}$ is proved in [1].

\section{FurTher QUESTIONS}

It seems likely that the following mixed characteristic analogue of Corollary [1.4 has a positive answer:

Question 4.1. let $K$ be a finite extension of $\mathbb{Q}_{p}, \mathcal{O}$ its ring of integers, $k$ the residue field and $X$ a smooth proper variety over $K$ such that $C H_{0}\left(X_{\overline{K(X)}}\right)_{\mathbb{Q}}=\mathbb{Q}$. If $\mathcal{X} \rightarrow \operatorname{Spec}(\mathcal{O})$ is a proper scheme with generic fibre isomorphic to $X$ and closed fibre $X_{0}$, then is $X_{0}(k) \neq \emptyset$ ?

From our proofs we do not obtain any information about the valuations of the eigenvalues of Frobenius acting on the étale cohomology of the fibres of $f$. One is thus lead to ask the following:

Question 4.2. Let $f: X \rightarrow Y$ be a proper dominant morphism of smooth irreducible varieties over a finite field $k$. Let $Z$ be the generic fibre of $f$ and assume that $C H_{0}\left(Z_{\overline{k(X)}}\right)_{\mathbb{Q}}=$ $\mathbb{Q}$. Then for all $y \in Y(k)$ and $i>0$, does $|k|$ divide all the eigenvalues of Frobenius acting on $H^{i}\left(\left(f^{-1}(y)\right)_{\bar{k}}, \mathbb{Q}_{l}\right)(l \neq \operatorname{char}(k))$ ?

Theorem 1.1 has the following Hodge theoretic analogue: 
Theorem 4.3. Let $f_{i}: X_{i} \rightarrow Y, i=1,2$, be proper dominant morphisms of smooth irreducible varieties over $\mathbb{C}$ and let $g: X_{1} \rightarrow X_{2}$ be a dominant morphism over $Y$. Let $Z_{i}$ be the generic fibre of $f_{i}$ and assume that $g_{*}: C H_{0}\left(Z_{1} \overline{\mathbb{C}\left(X_{1}\right)}\right)_{\mathbb{Q}} \rightarrow C H_{0}\left(Z_{2} \overline{\mathbb{C}\left(X_{1}\right)}\right)_{\mathbb{Q}}$ is an isomorphism. Then $\operatorname{gr}_{0}^{F}\left(\operatorname{Coker}\left(g^{*}: H_{c}^{i}\left(X_{1}, \mathbb{C}\right) \rightarrow H_{c}^{i}\left(X_{2}, \mathbb{C}\right)\right)\right)=0$ for all $i$, where $F$ denotes the Hodge filtration of Deligne.

The proof is essentially the same as that of Theorem 1.1 so we omit the details: one only needs to replace Lemma 2.4 by its Hodge theoretic counterpart.

The Hodge version of Question 4.2 is:

Question 4.4. Let $f: X \rightarrow Y$ be a proper dominant morphism of smooth irreducible varieties over $\mathbb{C}$. Let $Z$ be the generic fibre of $f$ and assume that $C H_{0}\left(Z_{\overline{\mathbb{C}(X)}}\right)_{\mathbb{Q}}=\mathbb{Q}$. Then for all $y \in Y(\mathbb{C})$ and $i>0$, is $g r_{0}^{F}\left(H^{i}\left(f^{-1}(y), \mathbb{C}\right)\right)=0$ ?

It seems likely, as was suggested to us by P. Brosnan, that there should be a purely motivic statement from which Theorems 1.2 and 4.3 should follow after taking realisations. The category of motives over a base of Corti and Hanamura [3] would seem to be a natural choice in which to formulate such a statement.

Remark 4.5. After this paper was submitted both Question 4.2 and (a stronger form of) Question 4.4 were shown to have positive answers by H. Esnault; her results appear in the appendix to this article [8]. A positive answer to Question 4.1 in case $\mathcal{X}$ is regular was also obtained by her in 9]; the results of that paper include a stronger version of Corollary [1.2 in the case that $Y$ is a curve.

Acknowledgements. N.F. thanks Arvind Nair for a remark which convinced him that Corollary 1.2 should be true (before Theorem 1.1] was proved) and Patrick Brosnan, Hélène Esnault, Madhav Nori and V. Srinivas for their comments on the results of this paper. He also thanks Hélène Esnault and Marc Levine for an invitation to visit the University of Essen in May-June 2003 where he was supported by the DFG and via the Wolfgang Paul prize program of the Humbolt Stiftung; lectures of Hélène Esnault that he attended at that time provided part of the motivation for the results and questions in this paper.

We also thank Bruno Kahn for his comments on the first version of this paper and for suggesting Theorem 1.1 as a common generalisation of Corollaries 1.2 and 1.3 .

\section{REFERENCES}

[1] S. Bloch, H. Esnault, And M. Levine, Decomposition of the diagonal and eigenvalues of Frobenius for Fano hypersurfaces, math.AG/0302109, to appear in Am. J. Math.

[2] F. Campana, Connexité rationnelle des variétés de Fano, Ann. Sci. École Norm. Sup. (4), 25 (1992), pp. $539-545$.

[3] A. Corti And M. Hanamura, Motivic decomposition and intersection Chow groups. I, Duke Math. J., 103 (2000), pp. 459-522.

[4] A. J. DE Jong, Smoothness, semi-stability and alterations, Inst. Hautes Études Sci. Publ. Math., (1996), pp. $51-93$.

[5] A. J. DE Jong, Families of curves and alterations, Ann. Inst. Fourier (Grenoble), 47 (1997), pp. 599621.

[6] P. Deligne and N. Katz, eds., Groupes de monodromie en géométrie algébrique. II, Springer-Verlag, Berlin, 1973. Séminaire de Géométrie Algébrique du Bois-Marie 1967-1969 (SGA 7 II), Lecture Notes in Mathematics, Vol. 340. 
[7] A. Doundy and J. Verdier, eds., Séminaire de Géométrie Analytique, Société Mathématique de France, Paris, 1976. Tenu à l'École Normale Supérieure, Paris, 1974-75, Astérisque, No. 36-37.

[8] H. Esnault, Appendix to "Congruences for rational points on varieties over finite fields" by N. Fakhruddin and C. S. Rajan, math.NT/0403265.

[9] — Deligne's integrality theorem in unequal characteristic and rational points over finite fields, math.NT/0405318.

[10] — Varieties over a finite field with trivial Chow group of 0-cycles have a rational point, Invent. Math., 151 (2003), pp. 187-191.

[11] W. Fulton, Intersection theory, Springer-Verlag, Berlin, second ed., 1998.

[12] M. J. Greenberg, Lectures on forms in many variables, W. A. Benjamin, Inc., New York-Amsterdam, 1969.

[13] B. KAHN, Number of points of function fields over finite fields, math.NT/0210202.

[14] N. M. Katz, On a theorem of Ax, Amer. J. Math., 93 (1971), pp. 485-499.

[15] J. Kollár, Y. Miyaoka, and S. Mori, Rational connectedness and boundedness of Fano manifolds, J. Differential Geom., 36 (1992), pp. 765-779.

School of Mathematics, Tata Institue of Fundamental Research, Homi Bhabha Road, Mumbai 400005, INDiA

E-mail address: naf@math.tifr.res.in, rajan@math.tifr.res.in 